\title{
Computation of Hopkins' 3-circle integrals using Zernike expansions
}

\author{
A. J. E. M. Janssen \\ a.j.e.m.janssen@tue.nl
}

Eindhoven University of Technology, Department EE and EURANDOM P.O. Box 513, 5600 MB Eindhoven, The Netherlands

The integrals occurring in optical diffraction theory under conditions of partial coherence have the form of an incomplete autocorrelation integral of the pupil function of the optical system. The incompleteness is embodied by a spatial coherence function of limited extent. In the case of circular optical systems and coherence functions supported by a disk, this gives rise to Hopkins' 3-circle integrals. In this paper, a computation scheme for these integrals (initially with coherence functions that are constant on their disks) is proposed where the required integral is expressed semi-analytically in the Zernike expansion coefficients of the pupil function. To this end, the Zernike expansion coefficients of a shifted pupil function restricted to the coherence disk are expressed in terms of the pupil function's Zernike expansion coefficients. Next, the required integral is expressed as an infinite series involving two sets of Zernike coefficients of restricted pupils using Parseval's theorem for orthogonal series. Due to a convenient separation of the radial parameters and the spatial variables, the method avoids a cumbersome administration involving separate consideration of various overlap situations. The computation method is extended to the case of coherence functions that are not necessarily constant on their supporting disks by using a result on linearization of the product of two Zernike circle polynomials involving Wigner coefficients. [DOI: http://dx.doi.org/10.2971/je0s.2011.11059]

Keywords: diffraction integral, partial coherence, Hopkins' 3-circle integrals, correlation, Zernike circle polynomials, Wigner coefficients

\section{INTRODUCTION AND OVERVIEW}

The calculation of the image intensity produced by an optical system, like a microscope or a projection lens, is mostly carried out via an analysis of the transfer of spatial frequencies from object to image plane. In this view the optical system acts as a low-pass filter. The first analysis in the frequencydomain goes back to Abbe, see [1], Sec. 8.6.3. (b), who studied the image formation in the case of a coherently illuminated object. He represented a general object with the aid of a periodic, grating-like structure. The concept of spatial frequency was introduced in optics by Duffieux, see [1], Sec. 9.5. He established the spatial frequency transfer function of an optical system when the object is incoherently illuminated and found it to be equal to the Fourier transform of the intensity point-spread function of the imaging system. Approximately a decade later, Hopkins [2] gave a detailed analysis of the frequency transfer by an optical system and the resulting modulation of the image intensity when the object is illuminated by a source of finite extent. In terms of coherence of object illumination, one speaks in this case of partial coherence. The spatial coherence function is neither unity (coherent) or a delta function (incoherent) but rather a complex function of the spatial coordinates that is bounded in modulus by its value at the origin.

From Abbe's theory, it is known that the frequency transfer function of a coherently illuminated object equals unity on the support of the circular pupil function. The spatial frequencies outside the pupil circle are lost for image formation and the transfer function vanishes there. For an incoherently illuminated object, the frequency transfer function is given accord- ing to Duffieux by the autocorrelation function of the pupil function. Hopkins general approach for the frequency transfer in the case of partial coherence requires the evaluation of the autocorrelation integral of the circular pupil function with integration area delimited by a third circle of different radius, see [2], [3]. For this reason, the term "3-circle overlap integral" is commonly used, also see [1], Sec. 10.6.3, and, in particular, Fig. 10.18. Numerical methods have generally been used to calculate these 3-circle overlap integrals, in the presence of an arbitrary transmittance and aberration of the pupil function of the imaging system.

In this paper, a semi-analytical expression for the value of Hopkins' 3-circle integral is proposed in terms of the expansion coefficients of the pupil function with respect to the orthogonal Zernike circle polynomials on the pupil circle. The pupil function and a shifted copy of it are restricted to the third disk and either restricted pupil function is expanded into the system of Zernike circle polynomials pertaining to the third disk in which the expansion coefficients are expressed in those of the pupil function on the pupil disk. For this an extension of a recent result, [4], Theorem 4.1, on the correlation of two circle polynomials is used. The required Hopkins integral then assumes, via Parseval's theorem for the inner product of two functions expanded into a set of orthogonal functions, the form of an infinite series involving the two sets of expansion coefficients and the normalization constants of the circle polynomials pertaining to the third disk. The required expansion coefficients, obtained via [4], Theorem 4.1 in terms of correlations of Zernike circle polynomials, also have the form of an 


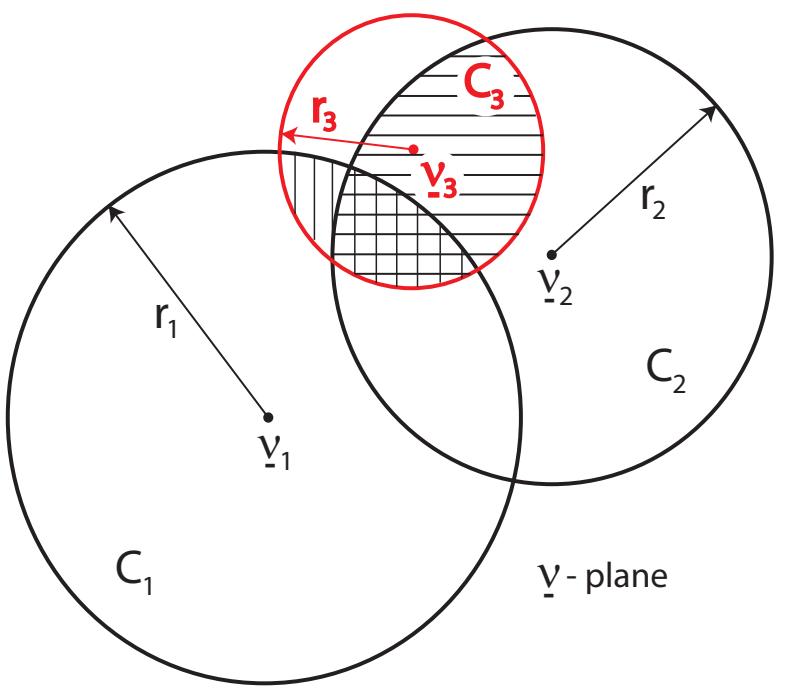

FIG. 1 Three circles $C_{a}$ in the $v$-plane, with general centers $v_{a}$ and radii $r_{a}$, $a=1,2,3$, where $C_{1}$ and $C_{2}$ support a generalized pupil function $F_{1}(v-v 1)$ and $F_{2}\left(v-v_{2}\right)$, respectively, and the third disk $C_{3}$ supports a transmittance $G\left(v-v_{3}\right)$ which is often constant on $C_{3}$. Supporting set of $Z_{0}^{0}(v) F_{1}\left(v-v_{1}\right)$ and of $Z_{0}^{0}(v) F_{2}\left(v-v_{2}\right)$ with vertical and horizontal hatching, respectively.

infinite series. An examination of the latter series reveals various symmetries, separation of radial parameters and cartesian variables, and opportunities to reuse results from previous calculations. This renders the new method a potentially attractive alternative to the more numerically oriented approaches. The convergence issues for both type of series that occur in the method are discussed by considering special, but realistic, cases that allow explicit analytic results from which one can tell what to expect in the general case. Finally, the method, initially proposed for the case of constant transmittance on the third disk, is extended for use with non-uniform transmittances. For this, a result is employed that linearizes the product of two Zernike polynomials on the third disk using Wigner or Clebsch-Gordan coefficients.

\section{MAIN RESULT AND COMPUTATION SCHEME}

Figure 1 shows three disks in the plane with centers $v_{a}$ and radii $r_{a}, a=1,2,3$, in a general overlap situation in which it is allowed that none of the three disks is contained in the overlap region of the other two. It is assumed that both disk 1 and 2 support a generalized pupil function (comprising phase and amplitude non-uniformities) $F_{1}$ and $F_{2}$. The integrals considered in this paper are of the form

$$
I=\int_{\left|\boldsymbol{v}-\boldsymbol{v}_{3}\right| \leq r_{3}} F_{1}\left(\boldsymbol{v}-\boldsymbol{v}_{1}\right) F_{2}^{*}\left(\boldsymbol{v}-\boldsymbol{v}_{2}\right) d \boldsymbol{v},
$$

i.e., an incomplete correlation integral of $F_{1}$ and $F_{2}$ in which the incompleteness is embodied by restriction of the integration to $v$ in the disk with center $v_{3}$ and radius $r_{3}$. Due to this limited integration range, a direct use of [4], Sec. 4 on the computation of the optical transfer function is not possible since there a full integration range is required. However, an educated use of [4], Theorem 4.1, together with Parseval's theo- rem for orthogonal series, leads to a semi-analytic result for the integral in Eq. (1). This will be elaborated below.

It is assumed without limitation of generality that $\boldsymbol{v}_{3}=\mathbf{0}$ and $r_{3}=1$. The pupil functions $F_{a}, a=1,2$, are assumed to be given in the form of Zernike series as

$$
F_{a}(\boldsymbol{v})=\sum_{n, m} \gamma_{n, a}^{m} Z_{n}^{m}\left(\boldsymbol{v} / r_{a}\right), \quad|\boldsymbol{v}| \leq r_{a},
$$

where the Zernike circle polynomials $Z_{n}^{m}$ vanish outside the unit disk and are given in complex notation as

$$
Z_{n}^{m}\left(\rho e^{i \vartheta}\right)=R_{n}^{|m|}(\rho) e^{i m \vartheta}, \quad 0 \leq \rho \leq 1,0 \leq \vartheta<2 \pi,
$$

with integer $n, m$ such that $n-|m|$ is non-negative and even and $R_{n}^{|m|}$ the radial polynomials. With $v_{3}=\mathbf{0}$ and $r_{3}=1$, the integral $I$ in Eq. (1) can be written as

$$
I=\iint Z_{0}^{0}(\boldsymbol{v}) F_{1}\left(\boldsymbol{v}-\boldsymbol{v}_{1}\right)\left(Z_{0}^{0}(\boldsymbol{v}) F_{2}\left(\boldsymbol{v}-\boldsymbol{v}_{2}\right)\right)^{*} d \boldsymbol{v},
$$

the circle polynomial $Z_{0}^{0}$ being constant 1 on the unit disk and 0 outside it. Assume now that the Zernike expansion

$$
Z_{0}^{0}(\boldsymbol{v}) F_{a}\left(\boldsymbol{v}-\boldsymbol{v}_{a}\right)=\sum_{n, m} \beta_{n, a}^{m}\left(\boldsymbol{v}_{a}\right) Z_{n}^{m}(\boldsymbol{v}), \quad|\boldsymbol{v}| \leq 1,
$$

of the pupil function $F_{a}$, shifted to center $v_{a}$ and restricted to the unit disk, is available. See Figure 1, where the supporting set of $Z_{0}^{0}(v) F_{1}\left(v-v_{1}\right)$ and that of $Z_{0}^{0}(v) F_{2}\left(v-v_{2}\right)$ have vertical and horizontal hatching, respectively, and the integration region for $I$ in Eq. (4) consists of the intersection of the two hatched regions. Then, by orthogonality of the $Z_{n}^{m}$ on the unit disk and the normalization

$$
\iint\left|Z_{n}^{m}(v)\right|^{2} d v=\frac{\pi}{n+1},
$$

there holds

$$
I=\sum_{n, m} \frac{\pi}{n+1} \beta_{n, 1}^{m}\left(\boldsymbol{v}_{1}\right)\left(\beta_{n, 2}^{m}\left(\boldsymbol{v}_{2}\right)\right)^{*} .
$$

The Zernike coefficients $\beta_{n, a}^{m}=\beta_{n, a}^{m}\left(\boldsymbol{v}_{a}\right)$ in Eq. (5) are given in integral form as

$$
\beta_{n, a}^{m}=\frac{n+1}{\pi} \iint Z_{0}^{0}(\boldsymbol{v}) F_{a}\left(\boldsymbol{v}-\boldsymbol{v}_{a}\right)\left(Z_{n}^{m}(\boldsymbol{v})\right)^{*} d \boldsymbol{v} .
$$

Inserting the Zernike expansion of Eq. (2), with summation variables $n^{\prime}, m^{\prime}$ rather than $n, m$, into Eq. (8) yields

$$
\beta_{n, a}^{m}=\frac{n+1}{\pi} \sum_{n^{\prime}, m^{\prime}} \gamma_{n^{\prime}, a}^{m^{\prime}}\left(Z_{n^{\prime}}^{m^{\prime}}\left(\cdot / r_{a}\right) * *_{\operatorname{corr}} Z_{n}^{m}\right)\left(-\boldsymbol{v}_{a}\right),
$$

where $* *_{\text {corr }}$ denotes correlation,

$$
\left(F * *_{\text {corr }} G\right)(\boldsymbol{v})=\iint F\left(\boldsymbol{v}^{\prime}+\boldsymbol{v}\right)\left(G\left(\boldsymbol{v}^{\prime}\right)\right)^{*} d \boldsymbol{v}^{\prime}, \quad \boldsymbol{v} \in \mathbb{R}^{2},
$$

of two square integrable functions $F, G$. The correlations as required in Eq. (9) are given by Theorem 2.1 below that generalizes [4], Theorem 4.1, which deals with the case $c^{\prime}=c$ in Eq.(11). This generalization is also relevant in the context of incoherent illumination when a pupil function, expanded on a full disk as a Zernike series, is partially obstructed by 
a, not necessarily centralized, subdisk as occurs in astronomical observation using mirror telescopes. Such a pupil function should be written as the difference of the unobstructed pupil function and the obstructed part of the full pupil function. The obstructed part has a Zernike series representation into circle polynomials pertaining to the obstructing disk, with coefficients that can be obtained from those of the unobstructed pupil function on the full disk via [4], Theorem 3.1. The autocorrelation function of the partially obstructed pupil function is then the sum of two auto-terms and two cross-terms, where the latter require Theorem 2.1 in its general form to produce an analytic result for the correlation function of two circle polynomials with reference disks of unequal radii.

Theorem 2.1. Let $n^{\prime}, m^{\prime}$ and $n, m$ be integers such that $n^{\prime}-\left|m^{\prime}\right|$ and $n-|m|$ are non-negative and even, and let $c^{\prime}, c>0$. Then for any $\xi^{\prime}, \eta^{\prime}$ and $\xi, \eta$ there holds

$$
\begin{aligned}
& \iint Z_{n^{\prime}}^{m^{\prime}}\left(\frac{\xi^{\prime}+v^{\prime \prime}}{c^{\prime}}, \frac{\eta^{\prime}+\mu^{\prime \prime}}{c^{\prime}}\right) \\
& \times\left(Z_{n}^{m}\left(\frac{\xi+v^{\prime \prime}}{c}, \frac{\eta+\mu^{\prime \prime}}{c}\right)\right)^{*} d v^{\prime \prime} d \mu^{\prime \prime} \\
= & \sum_{n^{\prime \prime}} C_{n^{\prime} n, n^{\prime \prime}}^{m^{\prime} m} Z_{n^{\prime \prime}}^{m^{\prime}-m}\left(\frac{\xi^{\prime}-\xi}{c^{\prime}+c}, \frac{\eta^{\prime}-\eta}{c^{\prime}+c}\right),
\end{aligned}
$$

with summation over all integer $n^{\prime \prime}$ such that $n^{\prime \prime}-\left(n^{\prime}+n\right)$ is non-negative and even, and where we identify $Z\left(\rho e^{i \vartheta}\right) \equiv Z(\nu, \mu)$, see Eq.(3), when $v+i \mu=\rho e^{i \vartheta}$. The $C^{\prime}$ s are given by

$$
\begin{aligned}
C_{n^{\prime} n, n^{\prime \prime}}^{m^{\prime} m}= & \left(\frac{c^{\prime} c}{c^{\prime}+c}\right)^{2} \frac{(-1)^{n}\left(n^{\prime \prime}+1\right) \pi}{\left(n^{\prime}+1\right)(n+1)} \\
& \times\left[S_{n^{\prime} n}^{n^{\prime \prime}+1}-S_{n^{\prime}+2, n}^{n^{\prime \prime}+1}-S_{n^{\prime}, n+2}^{n^{\prime \prime}+1}+S_{n^{\prime}+2, n+2}^{n^{\prime \prime}+1}\right]
\end{aligned}
$$

with

$$
\begin{aligned}
S_{i j}^{k+1}= & \frac{\left(\frac{1}{2}(k+i+j)\right) !\left(\frac{1}{2}(k-i-j)\right) !}{\left(\frac{1}{2}(k+i-j)\right) !\left(\frac{1}{2}(k+j-i)\right) !} \frac{\left(c^{\prime}\right)^{i} c^{j}}{\left(c^{\prime}+c\right)^{i+j}} \\
& \times\left(P_{\frac{1}{2}(k-i-j)}^{(i, j)}\left(\frac{c-c^{\prime}}{c+c^{\prime}}\right)\right)^{2}
\end{aligned}
$$

for integers $i, j, k \geq 0$ such that $k-i-j$ is non-negative and even, and $S_{i j}^{k+1}=0$ otherwise. The $P_{l}^{(\alpha, \beta)}(x)$ are the Jacobi polynomials, see [5], Ch. 22, of degree $l$ corresponding to the weight function $(1-x)^{\alpha}(1+x)^{\beta}$ on $[-1,1]$.

Proof. The proof consists of a repetition of the argument used to prove [4], Theorem 4.1, combined with the comments in Notes 1, 2 after the proof of [4], Theorem 4.1. Here the result of Bailey for the integral of the product of three Bessel functions is used as in [4], Sec. 6 to establish

$$
\int_{0}^{\infty} J_{i}\left(c^{\prime \prime} u a\right) J_{j}\left(c^{\prime \prime} u b\right) J_{k+1}\left(c^{\prime \prime} u\right) d u=\left(c^{\prime \prime}\right)^{-1}(-1)^{\frac{1}{2}(k-j)} S_{i j}^{k}
$$

with $c^{\prime \prime}=c^{\prime}+c, a=c / c^{\prime \prime}, b=c^{\prime} / c^{\prime \prime}$.

Thus the computation scheme for $I$ in Eq. (1) can be summarized as follows: $I$ is given by Eq. (7), where $\beta_{n, a}^{m}, a=1,2$, is computed from the Zernike expansion coefficients $\gamma_{n, a}^{m}$ of $F_{a}$ in Eq. (2) according to Eq. (9), in which the correlations of two
Zernike circle polynomials is given by Theorem 2.1, choice $c^{\prime}=1 / r_{a}, c=1,\left(\xi^{\prime}, \eta^{\prime}\right)=-v_{a},(\xi, \eta)=0$. Note that the method avoids a cumbersome administration with many instances, calling for separate consideration, as would occur in approaches based on numerical integration. In particular, the overlap situation of the three involved circles does not enter into the method.

\section{DISCUSSION OF THE MAIN RESULT AND COMPUTATION SCHEME}

\subsection{Comments on Theorem 2.1}

At the heart of the new computation method lies the result in Theorem 2.1 on the correlation of two circle polynomials. There is a number of obvious facts that follow from general properties of correlations $F * *$ corr $G$ of two functions $F$ and $G$ supported by disks $r_{F}$ and $r_{G}$, such as

$$
\begin{aligned}
F * *_{\text {corr }} G & =\left(G^{*} * *_{\text {corr }} F^{*}\right)^{*} ; \\
\left(F * *_{\text {corr }} G\right)(v) & =0,|v| \geq r_{F}+r_{G} .
\end{aligned}
$$

These results apply also to the correlation integral of two circle polynomials as given in Theorem 2.1. Note, for instance, that the right-hand side of Eq. (11) vanishes when $\left|\left(\xi-\xi^{\prime}, \eta^{\prime}-\eta\right)\right|>c^{\prime}+c$. There are the following special features.

3.1.1 The $C_{n^{\prime} n, n^{\prime \prime}}^{m^{\prime} m}$ do not depend on $m^{\prime}$ and $m$, except that they are used in Eq. (11) for ranges of $n^{\prime}, n$ and $n^{\prime \prime}$ that do depend on $m^{\prime}$ and $m$. Furthermore, the circle polynomials $Z$ at the right-hand side of Eq. (11) all have the same azimuthal order $m^{\prime}-m$. These two facts reduce the computational task considerably. For instance,

$$
Z_{n^{\prime}}^{m^{\prime}} * *_{\text {corr }} Z_{n}^{m}=Z_{n^{\prime}}^{m^{\prime}+2 k} * *_{\text {corr }} Z_{n}^{m+2 k}
$$

for all integer $k$ such that $\left|m^{\prime}+2 k\right| \leq n^{\prime},|m+2 k| \leq n$. For the case that $c^{\prime}, c, \xi^{\prime}, \eta^{\prime}, \xi, \eta$ are such that the support of one of the shifted-and-scaled Z's at the left-hand side of Eq. (11) is entirely contained in the support of the other, Theorem 3.1 in [4] gives a closed form result for the correlation integral in Eq. (11). This closed form result, however, does not extend to the general situation and involves both $m$ and $m^{\prime}$ as well as $c^{\prime}, c, \xi^{\prime}, \eta^{\prime}, \xi, \eta$ in a complicated and less transparent way.

3.1.2 The $C_{n^{\prime} n, n^{\prime \prime}}^{m^{\prime} m}$ depend on the radial parameters $c^{\prime}, c$ but not on the spatial variables $\xi^{\prime}, \eta^{\prime}, \xi, \eta$. Normally, in an application, the radial parameters are fixed while the spatial variables may vary freely. Hence, the same set of $C^{\prime}$ s can be used for all instances of spatial variables that occur in the application. Note also that the right-hand side of Eq. (11) depends on $\xi^{\prime}, \eta^{\prime}, \xi, \eta$ only through the differences $\xi^{\prime}-\xi, \eta^{\prime}-\eta$.

3.1.3 The circle polynomials at the right-hand side of Eq. (11) are required for potentially large degree $n^{\prime \prime}$. Then the direct form of the radial polynomials

$$
R_{n}^{m}(\rho)=\sum_{s=0}^{p}(-1)^{s} \frac{(n-s) ! \rho^{n-2 s}}{s !(p-s) !(q-s) !}
$$

with non-negative integers $n, m$ satisfying $n-m$ non-negative and even, and $p, q$ defined as $p=\frac{1}{2}(n-m), q=\frac{1}{2}(n+m)$, is 
cumbersome to use. However, there holds

$$
R_{n}^{m}(\rho)=\rho^{m} P_{p}^{(0, m)}\left(2 \rho^{2}-1\right),
$$

with $P_{l}^{(\alpha, \beta)}(x)$ the Jacobi polynomials. From the recursion relations that are satisfied by the Jacobi polynomials, the following recursive scheme for fixed $m=0,1, \ldots$ holds: $R_{m-2}^{m}(\rho)=0$, $R_{m}^{m}(\rho)=\rho^{m}$ and for $n=m+2, m+4, \ldots$

$$
\begin{aligned}
& \frac{(p+1)(q+1)}{(n+1)(n+2)} R_{n+2}^{m}(\rho) \\
= & \left(\rho^{2}-\frac{p(p+1)+q(q+1)}{n(n+2)}\right) R_{n}^{m}(\rho)-\frac{p q}{n(n+1)} R_{n-2}^{m}(\rho),
\end{aligned}
$$

see [6], Eq. (2.29). Alternatively, for any $n=0,1, \ldots$ and any $\rho, 0 \leq \rho \leq 1$, the values of all radial polynomials $R_{n}^{m}(\rho)$ with $m=n, n-2, \ldots, n-2\left\lfloor\frac{1}{2} n\right\rfloor=0$ or 1 can be computed using an algorithm of the DFT-type on $N(\geq 2 n+1)$ points, see [7]. The computation of the Jacobi polynomials $P_{l}^{(\alpha, \beta)}(x)$, as used in the expression in Eq. (13) for the $S$ 's, can also be done using recursion techniques.

3.1.4 As to the convergence of the series expression at the right-hand side of Eq. (11), there are the following comments. There holds

$$
\left|Z_{n}^{m}\left(\rho e^{i \vartheta}\right)\right| \leq 1, \quad 0 \leq \rho \leq 1, \quad 0 \leq \vartheta<2 \pi,
$$

while $\left|Z_{n}^{m}\left(\rho e^{i \vartheta}\right)\right|$ decays typically as $1 / n^{1 / 2}, n \rightarrow \infty$, when $m$ is fixed and $\rho$ is away from 0 and 1 . To assess decay behaviour of $C_{n^{\prime} n, n^{\prime \prime}}^{m^{\prime} m}$ as $n^{\prime \prime} \rightarrow \infty$, the special case that

$$
c^{\prime}=c=1, \quad n^{\prime}=m^{\prime}=n=m=0
$$

is considered. Then the function

$$
\begin{aligned}
& \left(Z_{0}^{0} * *_{\operatorname{corr}} Z_{0}^{0}\right)(\rho)=2\left(\arccos \frac{1}{2} \rho-\frac{1}{2} \rho \sqrt{1-\left(\frac{1}{2} \rho\right)^{2}}\right), \\
& 0 \leq \rho \leq 2,
\end{aligned}
$$

arises and

$$
\begin{aligned}
& C_{00,2 k}^{00}=\frac{\pi}{4}(2 k+1)\left[S_{0,0}^{2 k+1}-S_{2,0}^{2 k+1}-S_{0,2}^{2 k+1}+S_{2,2}^{2 k+1}\right], \\
& k=0,1, \ldots,
\end{aligned}
$$

are the quantities of interest. The $S$-quantities are expressed in terms of Jacobi polynomials with argument $x=\left(c-c^{\prime}\right) /\left(c+c^{\prime}\right)=0$ per Eq. (13), and a further elaboration, using the recursion properties in [8], Ch. V, (4.4), (4.16), yields

$$
\begin{aligned}
C_{00,4 l+2}^{00} & =-\frac{\pi}{4}(4 l+3) \frac{\left(P_{2 l}^{(0,0)}(0)\right)^{2}}{2(l+1)^{2}}, l=0,1, \ldots, \\
C_{00,4 l}^{00} & =\frac{\pi}{4}(4 l+1) \frac{\left(P_{2 l}^{(0,0)}(0)\right)^{2}}{2\left(l-\frac{1}{2}\right)(l+1)}, l=1,2, \ldots .
\end{aligned}
$$

Then from

$$
P_{2 l}^{(0,0)}(0)=(-1)^{l} \frac{\Gamma\left(l+\frac{1}{2}\right)}{\Gamma\left(\frac{1}{2}\right) \Gamma(l+1)} \approx \frac{(-1)^{l}}{\sqrt{\pi l}}, \quad l \rightarrow \infty,
$$

see [8], Ch. V, (10.17), it follows that $C_{00,2 k}^{00}=O\left(1 / k^{2}\right)$, $k \rightarrow \infty$. This indicates that one should expect a decay behaviour $1 /\left(n^{\prime \prime}\right)^{2}$ of the $C_{n^{\prime} n, n^{\prime \prime}}^{m^{\prime} m}$ as $n^{\prime \prime} \rightarrow \infty$. Hence, the series expansion in Eq. (11) for the correlation integral converges absolutely, but not particularly fast. However, from the asymptotics of the Jacobi polynomials of large degree, see [8], $\mathrm{Ch}$. V, (5.5), it follows that the terms in the series possess, asymptotically, much structure. For such series, various convergence acceleration techniques exist.

\subsection{Decay of $\beta$-coefficients}

The coefficients $\beta_{n, a}^{m}$ occur in the infinite series expression for $I$ in Eq. (7), and so it is of interest to have an idea how fast they decay to 0 as $|m| \leq n, n \rightarrow \infty$. It should be expected that this decay is not fast, the $\beta_{n, a}^{m}$ being Zernike coefficients of a discontinuous function. A special case is considered. For $r>0$, let

$$
F^{(r)}(\boldsymbol{v})= \begin{cases}1, & |v-(r, 0)| \leq r \\ 0, & \text { otherwise }\end{cases}
$$

Taking for $a=1,2$

$$
F_{a}(v)=\lim _{r \rightarrow \infty} F^{(r)}(v)= \begin{cases}1, & v=(\nu, \mu), v>0, \\ 0, & \text { otherwise },\end{cases}
$$

and restricting $F_{a}(v)$ to the unit disk $|v| \leq 1$, it is seen that the $\beta_{n, a}^{m}$ are the Zernike coefficients of the pupil function

$$
F(v, \mu)= \begin{cases}1, & v^{2}+\mu^{2} \leq 1, v>0, \\ 0, & \text { otherwise }\end{cases}
$$

There is for this case the special result

$$
F=\frac{1}{2} Z_{0}^{0}+\sum_{n, m} \frac{2}{\pi} i^{n-1} \frac{n+1}{n(n+2)} Z_{n}^{m}
$$

with summation over all integers $n, m$ such that $m$ is odd and $n-|m|$ is non-negative and even. Note that the coefficients $\beta_{n, a}^{m}$ do not depend explicitly on $m$, except that they are understood to vanish when $n<|m|$. The result in Eq. (30) follows from

$$
\beta_{n, a}^{m}=\frac{n+1}{\pi} \int_{-\pi / 2}^{\pi / 2} \int_{0}^{1} R_{n}^{|m|}(\rho) e^{-i m \vartheta} \rho d \rho d \vartheta
$$

and the explicit result

$$
\int_{0}^{1} R_{n}^{|m|}(\rho) \rho d \rho=\frac{(-1)^{\frac{n-|m|}{2}}|m|}{n(n+2)},
$$

see [9], Eq. (A2), case $\alpha=0$. Therefore, in this case, I can be evaluated from Eq. (7) by using summation variables $(n, m)=(0,0)$ and $n=2 k+1, k=0,1, \ldots$ and $|m|=2 l+1$, $l=0,1, \ldots, k$ as

$$
I=\frac{\pi}{4}+\sum_{k=0}^{\infty} \frac{\pi}{2 k+2}\left\{2 \sum_{l=0}^{k} \frac{4}{\pi^{2}}\left(\frac{2 k+2}{(2 k+1)(2 k+3)}\right)^{2}\right\}
$$

In this special case, where $F_{a}=F, a=1,2$, and $F^{2}=F$, see Eq. (29), there holds

$$
I=\int_{-\pi / 2}^{\pi / 2} \int_{0}^{1} \rho d \rho d \vartheta=\pi / 2
$$


a result that also can be established from Eq. (33) by some manipulations and using $\sum_{k=0}^{\infty}(2 k+1)^{-2}=\pi^{2} / 8$. The expression in \{\} at the right-hand side of Eq. (33) decays like $1 / k$. Thus, a decay behaviour

$$
\sum_{|m| \leq n} \beta_{n, 1}^{m}\left(\beta_{n, 2}^{m}\right)^{*}=O\left(\frac{1}{n}\right)
$$

should be expected. Returning to Eq. (7) for $I$, it is seen that there is absolute convergence for the infinite series over $n$, although the convergence is rather slow. Again, convergence acceleration techniques should be considered.

\subsection{Zernike representation of pupil functions}

The expression for $\beta_{n, a}^{m}$ in Eq. (9) also involves the Zernike expansion coefficients $\gamma_{n^{\prime}, a}^{m^{\prime}}$ of the pupil functions $F_{a}, a=1,2$, on their pupil circles. Normally, these pupil functions are smooth and only a limited number of $\gamma^{\prime}$ s is required in the Zernike expansion.

\section{EXTENSION TO NON-UNIFORM TRANSMITTANCE}

Until now, the attention has been limited to the case that the integration over the third disk is performed with a uniform weight function. Image enhancement methods, for instance those used in optical lithography, successfully exploit the effect of a spatial modulation of the source intensity distribution. This can be done by adding new, spatially separated sources that can be handled with the analysis given so far. Other image enhancement approaches require a modulation of the source intensity within the circumscribing disk. To cover such advanced illumination methods in our analysis, we replace $I$ of Eq. (1) by $\left(\boldsymbol{v}_{3}=\mathbf{0}\right.$ and $r_{3}=1$, as earlier)

$$
I=\iint_{|\boldsymbol{v}| \leq 1} G(\boldsymbol{v}) F_{1}\left(\boldsymbol{v}-\boldsymbol{v}_{1}\right) F_{2}^{*}\left(\boldsymbol{v}-\boldsymbol{v}_{2}\right) d \boldsymbol{v},
$$

where $G$ is a smooth non-uniformity vanishing outside the unit disk. Then, compare Eqs. (4)-(5) for the case $a=1$, the Zernike expansion of $G(v) F_{1}\left(v-v_{1}\right)$ rather than that of $Z_{0}^{0}(v) F_{1}\left(v-v_{1}\right)$ is required. From the Zernike expansions

$$
G(v)=\sum_{j, i} \gamma_{j}^{i} Z_{j}^{i}(v)
$$

and

$$
Z_{0}^{0}(\boldsymbol{v}) F_{1}\left(\boldsymbol{v}-\boldsymbol{v}_{1}\right)=\sum_{l, k} \beta_{l, 1}^{k} Z_{l}^{k}(\boldsymbol{v}) ，
$$

see Eq. (5), it follows that

$$
G(\boldsymbol{v}) F_{1}\left(\boldsymbol{v}-\boldsymbol{v}_{1}\right)=\sum_{j, i, l, k} \gamma_{j}^{i} \beta_{l, 1}^{k} Z_{j}^{i}(\boldsymbol{v}) Z_{l}^{k}(\boldsymbol{v}) .
$$

When $G$ is a smooth non-uniformity, only a few terms are required in the expansion in Eq. (37).

The Zernike expansion of $G(\boldsymbol{v}) F_{1}\left(\boldsymbol{v}-\boldsymbol{v}_{1}\right)$ can be obtained from Eq. (39) when there is a (systematic) way to write

$$
Z_{j}^{i} Z_{l}^{k}=\sum_{n} A_{j l n}^{i k m} Z_{n}^{m}
$$

with $m=i+k$, as a linear combination of circle polynomials $Z_{n}^{m}$. For this, the following result is given in [10], Eq. (22). Let $j, i$ and $l, k$ be two pairs of integers such that $j-|i|$ and $l-|k|$ are even and non-negative. Then (40) holds with $m=i+k$ and with summation range

$$
n=\max (|i+k|,|j-l|)(2)(j+l),
$$

where $a(2) b$ denotes $a, a+2, \ldots, b$ when $b-a$ is non-negative and even. The $A_{j l n}^{i k m}$ are defined as follows. Let

$$
\begin{aligned}
& s=\frac{j-i}{2}, \quad t=\frac{j+i}{2}, \quad u=\frac{l-k}{2} \\
& v=\frac{l+k}{2}, \quad p=\frac{n-m}{2}, \quad q=\frac{n+m}{2},
\end{aligned}
$$

where $n$ is an integer such that $n-|m|$ is even and nonnegative. Then $A$ is given in terms of Wigner or ClebschGordan coefficients as

$$
A_{j l n}^{i k m}=\left(C_{\frac{1}{2} i, \frac{1}{2} k, \frac{1}{2} m}^{\frac{1}{2} j, \frac{1}{2} l, \frac{1}{2} n}\right)^{2}=\left|\left(\frac{1}{2} j \frac{1}{2} l \frac{1}{2} i \frac{1}{2} k \mid \frac{1}{2} j \frac{1}{2} l \frac{1}{2} n \frac{1}{2} m\right)\right|^{2},
$$

see [10], Eq. 22 (where $\langle\mid\rangle$ is used instead of $(\mid)$ in the third member of Eq. (43)) and [5], 27.9.1. In the notations introduced in Eq. (42), there holds, see [11], (3.170)

$$
\begin{aligned}
A_{j l n}^{i k m}= & \frac{(n+1)(s+u-p) !(i+s+p-u) !(k+u+p-s) !}{(i+k+s+u+p+1) !} \\
& \cdot s ! t ! u ! v ! p ! q ! \\
& \cdot\left(\sum_{z} \frac{(-1)^{z}}{z !(s+u-p-z) !(s-z) !(v-z) !}\right. \\
& \left.\times \frac{1}{(i+p-u+z) !(p-s+z) !}\right)^{2}
\end{aligned}
$$

when $n$ is as in Eq. (41), and $A_{j l n}^{i k m}=0$ otherwise. When $n$ is as in Eq. (41), all factorials in Eq. (44) in front of $\left(\sum_{z}\right)^{2}$ have a non-negative argument. Furthermore, in $\sum_{z}$ those integer $z$ are included for which all factorials involved in the $z^{\text {th }}$ term have a non-negative argument, and the summation range thus obtained is non-empty when $n$ is as in Eq. (41).

\section{CONCLUSION AND OUTLOOK}

There has been presented a semi-analytical method to compute the 3-circle integrals of Hopkins from the Zernike expansion coefficients of the involved generalized pupil functions on their supporting disks. The key ingredients are Parseval's theorem for the inner product of two functions expanded as orthogonal series and an explicit, infinite-series expression for the correlation of two Zernike circle polynomials on reference disks of general radii. The latter correlations can be evaluated in the form of a Zernike expansion on the disk whose radius is the sum of the two radii of the two reference disks, with explicit expansion coefficients having various symmetry and invariance properties. Convergence behaviour of the infinite series that arise in the method has been assessed by considering special cases that allow analytic treatment. The method allows extension to the case of non-uniform coherence functions on the extent of the illumination source.

The new method is a potential alternative for more numerically oriented methods to compute the 3-circle integrals. It 
is envisaged that for this the inherent symmetries and invariances are very helpful and that convergence acceleration techniques, taking account of the structure of the terms in the various infinite series, should be employed. At present, there has been done some numerical work on validation of Theorem 2.1, but otherwise there is only limited insight as to how the method compares to more numerically oriented approaches in terms of computation time and accuracy.

\section{ACKNOWLEDGEMENT}

The author wishes to thank Dr. S. van Haver for fruitful discussions.

\section{References}

[1] M. Born, and E. Wolf, Principles of Optics (7 $7^{\text {th }}$ ed., Cambridge University Press, Cambridge, 1999).

[2] H. H. Hopkins, "On the diffraction theory of optical images" P. R. Soc. A 217, 408-432 (1953).

[3] H. H. Hopkins, "Diffraction theory of laser read-out systems for optical video discs" J. Opt. Soc. Am. 69, 4-24 (1979).

[4] A. J. E. M. Janssen, "New analytic results for the Zernike circle polynomials from a basic result in the Nijboer-Zernike diffraction theory" J. Eur. Opt. Soc.-Rapid 6, 11028 1-14 (2011).

[5] M. Abramowitz, and I. A. Stegun, Handbook of Mathematical Functions (Dover Publications, New York, 1972).

[6] B. R. A. Nijboer, The Diffraction Theory of Aberrations (Ph. D. thesis, University of Groningen, 1942).
[7] A. J. E. M. Janssen, and P. Dirksen, “Computing Zernike polynomials of arbitrary degree using the discrete Fourier transform" J. Eur. Opt. Soc.-Rapid 2, 07012 1-3 (2007).

[8] F. G. Tricomi, Vorlesungen über Orthogonalreihen (Springer-Verlag, Berlin, 1955).

[9] J. J. M. Braat, P. Dirksen, A. J. E. M. Janssen, and A. S. van de Nes, "Extended Nijboer-Zernike representation of the vector field in the focal region of an aberrated high-aperture optical system" J. 0pt. Soc. Am. A 20, 2281-2292 (2003).

[10] W. J. Tango, "The circle polynomials of Zernike and their application in optics" Appl. Phys. 13, 327-332 (1977).

[11] L. C. Biedenharn, and J. D. Louck, Angular Momentum in Quantum Physics (Addison-Wesley, Reading, Massachusetts, 1981). 